\title{
A COEFFICIENT INEQUALITY FOR CERTAIN CLASSES OF ANALYTIC FUNCTIONS
}

\section{R.M.GOEL AND BEANT SINGH MEHROK}

\begin{abstract}
Let $f(z)=z+\sum_{k=2}^{\infty} a_{k} z^{k}$ be analytic in the unit disc $E=\{z:|z|<1\}$. We wish to maximize $\left|a_{3}-u a_{2}^{2}\right|$ over certain classes of analytic functions defined by convex subordination. This paper is concerned with the solution of the above extremal problem over certain classes of univalent analytic functions.
\end{abstract}

\section{Introduction}

Let $U$ denote the class of functions

$$
w(z)=\sum_{k=1}^{\infty} c_{k} z^{k}
$$

which are analytic in $E=\{z:|z|<1\}$ and satisfying there the conditions $w(0)=0$ and $|w(z)|<1$.

Let $S$ denote the class of functions

$$
f(z)=z+\sum_{k=2}^{\infty} a_{k} z^{k}
$$

analytic and univalent in $E$.

Let $K$ and $S^{*}$ be the sub-classes of $S$ which are, respectively convex and starlike in $E$. We shall call the function $f(z)$ of the class $S$ an alpha-convex ( $\alpha$-convex) function if

$$
\begin{aligned}
& \text { (i) } \frac{f(z) f^{\prime}(z)}{z} \neq 0 \text { and for any real } \alpha \\
& \text { (ii) } \operatorname{Re}\left[(1-\alpha) \frac{z f^{\prime}(z)}{f(z)}+\alpha\left(1+z \frac{f^{\prime \prime}(z)}{f^{\prime}}\right)\right]>0, z \in E \text {. }
\end{aligned}
$$

1980 Mathematics subject classification (Amer. Math. Soc.) 30 A 32, 30 A 34, 30 A 42.

Keywords and phrases : Subordination, convolution (Hadamard product), sub-classes of univalent analytic functions, extremal functions. 
Mocanu [15] introduced the concept of $\alpha$-convex functions. Miller, Mocanu and Reade [14] have shown that $\alpha$-convex functions are starlike in $E$, and for $\alpha \geq 1$, all $\alpha$ convex functions are convex in $E$. Therefore $\alpha$-convex functions are also called $\alpha$-starlike functions. Concept of $\alpha$-convex functions gives a continuous parametrization between starlike functions and convex function.

Al-Amiri and Read [1] introduced the class $H(\alpha)$ of analytic functions $f(z)$ in $E$ which satisfies the condition

$$
\operatorname{Re}\left[(1-\alpha) f^{\prime}(z)+\alpha\left(1+z \frac{f^{\prime \prime}(z)}{f^{\prime}(z)}\right)\right]>0 .
$$

Let $B(\alpha, \beta, p, g) \equiv B$ denote the class of functions $f(z)$ regular in $E$ and defined for $\beta$ real and $\alpha>0$ by

$$
f(z)=\left[(\alpha+i \beta) \int_{0}^{z} p(t) t^{\beta-1} g^{\alpha}(t) d t\right]^{\frac{1}{(\alpha+i \beta)}}
$$

where $p(z)$ is regular, $p(0)=1$ and $\operatorname{Re} p(z)>0$, and $g \in S^{*}$. (The powers appearing in (1.3) are principal values).

Bazilevic [2] showed that functions of the class $B$ are univalent in $E$. ditions

Let $M(\alpha ; A, B)$ be the class of functions $f(z)$ analytic in $E$ and satisfying the con-

$$
\begin{gathered}
\frac{f(z) f^{\prime}(z)}{z} \neq 0 \quad \text { and for } \quad \alpha \geq 0, \\
(1-\alpha) \frac{z f^{\prime}(z)}{f(z)}+\alpha\left(1+z \frac{f^{\prime \prime}(z)}{f^{\prime}(z)}\right)<\frac{1+A z}{1+B z}, \quad-1 \leq B<A \leq 1 .
\end{gathered}
$$

To avoid repetition, we lay down, once for all that $-1 \leq B<A \leq 1, \alpha \geq 0$ and $z \in E$.

Let $H(\alpha ; A, B)$ denote the class of functions $f(z)$ analytic in $E$ and satisfying the condition

$$
(1-\alpha) f^{\prime}(z)+\alpha\left(1+z \frac{f^{\prime \prime}(z)}{f^{\prime}(z)}\right)<\frac{1+A z}{1+B z} .
$$

If we take $\beta=0$ and $g(z) \equiv z$ in (1.3),

$$
\begin{aligned}
B_{1}(\alpha) & =B(\alpha, 0, p, z) \quad \text { is the class of functions } \\
f(z) & =\left[\alpha \int_{0}^{z} p(t) t^{\alpha-1} d t\right]^{\frac{1}{\alpha}}
\end{aligned}
$$

The class $B_{1}(\alpha)$ was defined by Singh in [16], and studied by Thomas in [18] and ElAshwah and Thomas in $[4,5]$.

Let $B_{1}(\alpha ; A, B)$ be a sub-class of Bazilevic functions such that

$$
\frac{z f^{\prime}(z)(f(z))^{\alpha-1}}{z^{\alpha}}<\frac{1+A z}{1+B z} .
$$


Let $F(\alpha ; A, B)$ and $G(\alpha ; A, B)$ denote the classes of functions $f(z)$ analytic in $E$ and satisfying respectively, the conditions

$$
f^{\prime}(z)+\alpha z f^{\prime \prime}(z)<\frac{1+A z}{1+B z}
$$

and

$$
(1-\alpha) \frac{f(z)}{z}+\alpha f^{\prime}(z)<\frac{1+A z}{1+B z}
$$

$f \in F(\alpha ; A, B)$ if and only if $z f^{\prime}(z) \in G(\alpha ; A, B)$.

Fekete and Szegö [6] made an early study for the estimates of $\left|a_{3}-\mu a_{2}^{2}\right|$ when $f(z)$ is analytic and univalent in $E$. The well-known result due to them states that if $f(z)$ is analytic univalent in $E$, then

$$
\left|a_{3}-\mu a_{2}^{2}\right| \leq \begin{cases}4 \mu-3, & \mu \geq 1 \\ 1+2 \exp \left(\frac{-2 \mu}{1-\mu}\right), & 0 \leq \mu \leq 1 \\ 3-4 \mu, & \mu \leq 0\end{cases}
$$

Hummel $[10,11]$ proved the conjecture of V.Singh that $\left|a_{3}-a_{2}^{2}\right| \leq \frac{1}{3}$ for the class $K$ of convex functions. Keogh and Merkes [13] obtained sharp estimates for $\left|a_{3}-\mu a_{2}^{2}\right|$ when $f$ is close-to-convex, starlike and convex in $E$. For $\mu$ complex Szynal [17] ined the estimates for $\left|a_{3}-\mu a_{2}^{2}\right|$ for the Mocanu class $M(\alpha)$. Al-Amiri and Reade [1] obtained estimates for $\left|a_{3}-\mu a_{2}^{2}\right|$ for the class $H(\alpha)$ when $\mu$ is complex. Singh [16] obtained the estimates for $\left|a_{3}-\mu a_{2}^{2}\right|$ for the class $B_{1}(\alpha)$.

The following observations are obvious.

(i) $M(\alpha ; 1,-1) \equiv M(\alpha)$, class of $\alpha$-convex functions,

(ii) $M(0 ; A, B) \equiv B_{1}(0 ; A, B) \equiv S^{*}(A, B)$, a sub-class of starlike functions studied by Janowski [12], and the authors [7] obtained some coefficient estimates for the class $S^{*}(A, B)$

(iii) $M(1 ; A, B) \equiv K(A, B)$, a sub-class of convex functions studied by the authors in [8], (However, estimates of $\left|a_{3}-\mu a_{2}^{2}\right|$ for the classes $S^{*}(A, B)$ and $K(A, B)$ remained unproved)

(iv) $M(0 ; 1,-1) \equiv B_{1}(0 ; 1,-1) \equiv S^{*}$,

(v) $M(1 ; 1,-1) \equiv H(1 ; 1,-1) \equiv K$,

(vi) $H(\alpha ; 1,-1) \equiv H(\alpha)$,

(vii) $H(0 ; A, B) \equiv B_{1}(1 ; A, B) \equiv R(A, B)$, a sub-class of univalent analytic functions studied by the authors in [9],

(viii) $F(\alpha ; 1,-1) \equiv F(\alpha)$ and $G(\alpha ; 1,-1) \equiv G(\alpha)$, classes considered by Chichra in [3];

(ix) $H(0 ; 1,-1) \equiv B_{1}(1 ; 1,-1) \equiv F(0 ; 1,-1) \equiv G(1 ; 1,-1) \equiv R$. $R$ is the NoshiroWarschawski class studied by several authors.

In this paper, we obtain sharp estimates for $\left|a_{3}-\mu a_{2}^{2}\right|$ when $f \in M(\alpha ; A, B)$ or $H(\alpha ; A, B)$ or $F(\alpha ; A, B)$ or $G(\alpha ; A, B)$ or $B_{1}(\alpha ; A, B)$. 
Results due to Keogh and Merkes [13], Szynal [17], Al-Amiri and Reads [1], and Singh [16] follow as special cases from our theorems.

2. Coefficient inequality

Theorem 2.1. If $f \in M(\alpha ; A, B)$, then

(i) for $\mu$ complex

$$
\left|a_{3}-\mu a_{2}^{2}\right| \leq \begin{cases}\frac{(A-B)}{2(1+2 \alpha)}, & |\mu-\gamma| \leq v \\ \frac{(A-B)^{2}}{(1+\alpha)^{2}}|\mu-\gamma|, & |\mu-\gamma| \geq v\end{cases}
$$

and

(ii) for $\mu$ real,

$$
\left|a_{3}-\mu a_{2}^{2}\right| \leq \begin{cases}\frac{(A-B)^{2}}{(1+\alpha)^{2}}(\gamma-\mu), & \mu \leq \gamma-v \\ \frac{(A-B)}{2(1+2 \alpha)}, & \gamma-v \leq \mu \leq \gamma+v \\ \frac{(A-B)^{2}}{(1+\alpha)^{2}}(\mu-\gamma), & \mu \geq \gamma+v\end{cases}
$$

where

$$
\begin{gathered}
\gamma=\frac{(A-B)(1+3 \alpha)-B(1+\alpha)^{2}}{2(1+2 \alpha)(A-B)} \\
v=\frac{(1+\alpha)^{2}}{2(1+2 \alpha)(A-B)}
\end{gathered}
$$

All the estimates are sharp.

Proof. From (1.4), by definition of subordination,

$$
(1-\alpha) \frac{z f^{\prime}(z)}{f(z)}+\alpha\left(1+z \frac{f^{\prime \prime}(z)}{f^{\prime}(z)}\right)=\frac{1+A w(z)}{1+B w(z)} .
$$

By expanding (2.8) and equating the coefficients we have

$$
a_{2}=\frac{(A-B) c_{1}}{1+\alpha}
$$

and

$$
a_{3}=\frac{(A-B)}{2(1+2 \alpha)} c_{2}+\left[\frac{(1+3 \alpha) a_{2}^{2}-B(A-B) c_{1}^{2}}{2(1+2 \alpha)}\right]
$$


From (2.9) and (2.10), we get

$$
a_{3}-\mu a_{2}^{2}=\frac{(A-B)}{2(1+2 \alpha)} c_{2}+\frac{(A-B)^{2}}{(1+\alpha)^{2}}\left[\frac{(A-B)(1+3 \alpha)-B(1+\alpha)^{2}}{2(1+2 \alpha)(A-B)}-\mu\right] c_{1}^{2} \text {. }
$$

Therefore

$$
\left|a_{3}-\mu a_{2}^{2}\right| \leq \frac{(A-B)}{2(1+2 \alpha)}\left|c_{2}\right|+\frac{(A-B)^{2}}{(1+\alpha)^{2}}|\gamma-\mu|\left|c_{1}\right|^{2} .
$$

Also

$$
\begin{gathered}
\left|c_{2}\right| \leq 1-\left|c_{1}\right|^{2} \\
\left|c_{1}\right| \leq 1
\end{gathered}
$$

(2.11) and (2.12) lead us to

$$
\left|a_{3}-\mu a_{2}^{2}\right| \leq \frac{(A-B)}{2(1+\alpha)}+\frac{(A-B)^{2}}{(1+\alpha)^{2}}[|\gamma-\mu|-v]\left|c_{1}\right|^{2} .
$$

If $|\gamma-\mu| \leq v=\frac{(1+\alpha)^{2}}{2(1+2 \alpha)(A-B)},(2.1)$ follows.

If $|\gamma-\mu| \geq v=\frac{(1+\alpha)^{2}}{2(1+2 \alpha)(A-B)},(2.2)$ follows, since $\left|c_{1}\right| \leq 1$.

Consider the case when $\mu$ is real.

Case I. $\quad \mu \leq \gamma$.

From (2.14), we have

$$
\left|a_{3}-\mu a_{2}^{2}\right| \leq \frac{(A-B)}{2(1+2 \alpha)}+\frac{(A-B)^{2}}{(1+\alpha)^{2}}[(\gamma-v)-\mu]\left|c_{1}\right|^{2} .
$$

If $\mu \leq(\gamma-v)$, from (2.15) we have

$$
\begin{aligned}
\left|a_{3}-\mu a_{2}^{2}\right| & \leq \frac{(A-B)}{2(1+2 \alpha)}+\frac{(A-B)^{2}}{(1+\alpha)^{2}}(\gamma-\mu-v), \text { since }\left|c_{1}\right| \leq 1 \\
& =\frac{(A-B)^{2}}{(1+\alpha)^{2}}(\gamma-\mu) .
\end{aligned}
$$

If $\gamma-v \leq \mu \leq \gamma$, we obtain (2.4) from (2.15).

\section{Case III. $\quad \mu \geq \gamma$.}

From (2.14) we have

$$
\left|a_{3}-\mu a_{2}^{2}\right| \leq \frac{(A-B)}{2(1+2 \alpha)}+\frac{(A-B)^{2}}{(1+\alpha)^{2}}[\mu-\gamma-v]\left|c_{1}\right|^{2},\left|c_{1}\right| \leq 1 .
$$


If $\mu \leq \gamma+v$, (2.4) follows. If $\mu \geq \gamma+v$, we obtain (2.5) from (2.16).

The estimates (2.1) and (2.4) are sharp for the function $f_{0(1)}(z)$ defined by

$$
f_{0(1)}(z)=\left\{\begin{array}{lll}
{\left[\frac{1}{\alpha} \int_{0}^{z} \frac{1}{\alpha}-1\left(1+B \delta t^{2}\right)^{\frac{(A-B)}{2 \alpha B}} d t\right]^{\alpha},} & B \neq 0, & |\delta|=1 \\
{\left[\frac{1}{\alpha} \int_{0}^{z} \frac{1}{\alpha}-1 \exp \left(\frac{A \delta t^{2}}{2 \alpha}\right) d t\right]^{\alpha},} & B=0 . &
\end{array}\right.
$$

The estimates (2.2), (2.3), (2.5) are sharp for the function $f_{1(1)}(z)$ defined by

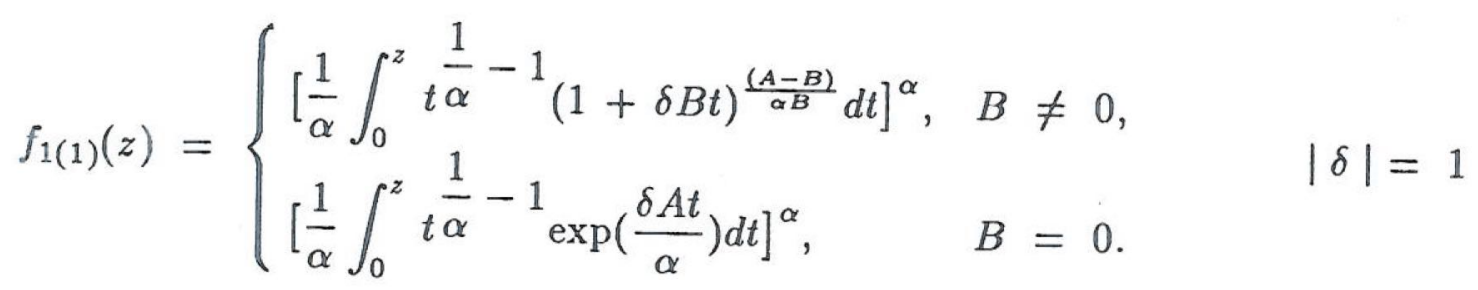

On the same lines we have

Theorem 2.2. Let $f \in H(\alpha ; A, B)$, then

(i) for $\mu$ complex,

$$
\left|a_{3}-\mu a_{2}^{2}\right| \leq \begin{cases}\frac{(A-B)}{3(1+\alpha)}, & :\left|\gamma_{1}-\mu\right| \leq \frac{4}{3(1+\alpha)(A-B)} \\ \frac{(A-B)^{2}}{4}\left|\gamma_{1}-\mu\right|, & \left|\gamma_{1}-\mu\right| \geq \frac{4}{3(1+\alpha)(A-B)}\end{cases}
$$

and

(ii) for $\mu$ real,

$$
\begin{aligned}
& \left|a_{3}-\mu a_{2}^{2}\right| \\
\leq & \begin{cases}\frac{(A-B)^{2}}{4}\left(\gamma_{1}-\mu\right), & \mu \leq \frac{4[\alpha(A-B)-(1+B)]}{3(1+\alpha)(A-B)} \\
\frac{(A-B)}{3(1+\alpha)}, & \frac{4[\alpha(A-B)-(1+B)]}{3(1+\alpha)(A-B)} \leq \mu \leq \frac{4[\alpha(A-B)+(1-B)]}{3(1+\alpha)(A-B)} \\
\frac{(A-B)^{2}}{4}\left(\mu-\gamma_{1}\right), & \mu \geq \frac{4[\alpha(A-B)+(1-B)]}{3(1+\alpha)(A-B)}\end{cases}
\end{aligned}
$$

where

From (1.5), we have

$$
\gamma_{1}=\frac{4[\alpha A-(1+\alpha) B]}{3(1+\alpha)(A-B)}
$$

$$
(1-\alpha) f^{\prime}(z)+\alpha\left(1+z \frac{f^{\prime \prime}(z)}{f^{\prime}(z)}\right)=\frac{1+A w(z)}{1+B w(z)}
$$


Bounds (2.17) and (2.20) are sharp for $w(z)=z^{2}$. (2.18), (2.19) and (2.21) are sharp for $w(z)=z$.

Theorem 2.3. Let $f \in B_{1}(\alpha ; A, B)$, then

(i) for any complex number $\mu$,

$$
\left|a_{3}-\mu a_{2}^{2}\right| \leq \begin{cases}\frac{(A-B)}{2+\alpha},\left|\gamma_{2}-\mu\right| \leq \frac{(1+\alpha)^{2}}{(2+\alpha)(A-B)} \\ \frac{(A-B)^{2}}{(1+\alpha)^{2}}\left|\gamma_{2}-\mu\right|, \quad\left|\gamma_{2}-\mu\right| \geq \frac{(1+\alpha)^{2}}{(2+\alpha)(A-B)}\end{cases}
$$

and

(ii) for any real number $\mu$,

$$
\left|a_{3}-\mu a_{2}^{2}\right| \leq \begin{cases}\frac{(A-B)^{2}}{(1+\alpha)^{2}}\left(\gamma_{2}-\mu\right), & \mu \leq \frac{(1-\alpha)(2+\alpha)(A-B)-2(1+B)(1+\alpha)^{2}}{2(2+\alpha)(A-B)} \\ \frac{(A-B)}{2+\alpha}, & \frac{(1-\alpha)(2+\alpha)(A-B)-2(1+B)(1+\alpha)^{2}}{2(2+\alpha)(A-B)} \\ \frac{(A-B)^{2}}{(1+\alpha)^{2}}\left(\mu-\gamma_{2}\right), & \mu \geq \frac{(1-\alpha)(2+\alpha)(A-B)+2(1-B)(1+\alpha)^{2}}{2(2+\alpha)(A-B)}\end{cases}
$$

where

$$
\gamma_{2}=\frac{(1-\alpha)(2+\alpha)(A-B)-2 B(1+\alpha)^{2}}{2(2+\alpha)(A-B)} .
$$

Equality signs in (2.22) and (2.25) hold for the function

$$
f_{0(2)}(z)=\left[\alpha \int_{0}^{z} t^{\alpha-1}\left(\frac{1+A \delta t^{2}}{1+B \delta t^{2}}\right) d t\right]^{\frac{1}{\alpha}}, \quad|\delta|=1 .
$$

Equality signs in (2.23), (2.24) and (2.26) are attained by the function

$$
f_{1(2)}(z)=\left[\alpha \int_{0}^{z} t^{\alpha-1}\left(\frac{1+A \delta t}{1+B \delta t}\right) d t\right]^{\frac{1}{\alpha}}, \quad|\delta|=1 .
$$

Theorem 2.4. If $f \in F(\alpha ; A, B)$, then

(i) for $\mu$ complex,

$$
\left|a_{3}-\mu a_{2}^{2}\right| \leq \begin{cases}\frac{(A-B)}{3(1+2 \alpha)}, & \left|\mu+\gamma_{3}\right| \leq \frac{4(1+\alpha)^{2}}{3(A-B)(1+2 \alpha)} \\ \frac{(A-B)^{2}}{4(1+\alpha)^{2}}\left|\mu+\gamma_{3}\right|, & \left|\mu+\gamma_{3}\right| \geq \frac{4(1+\alpha)^{2}}{3(A-B)(1+2 \alpha)}\end{cases}
$$

and 
(ii) for $\mu$ real

$$
\begin{aligned}
& \quad\left|a_{3}-\mu a_{2}^{2}\right| \\
& \leq \begin{cases}\frac{-(A-B)^{2}}{4(1+\alpha)^{2}}\left(\mu+\gamma_{3}\right), & \mu \leq \frac{-4(1+B)(1+\alpha)^{2}}{3(A-B)(1+2 \alpha)}, \\
\frac{(A-B)}{3(1+2 \alpha)}, & \frac{-4(1+B)(1+\alpha)^{2}}{3(A-B)(1+2 \alpha)} \leq \mu \leq \frac{4(1-B)(1+\alpha)^{2}}{3(A-B)(1+2 \alpha)}, \\
\frac{(A-B)^{2}}{4(1+\alpha)^{2}}\left(\mu+\gamma_{3}\right), & \mu \geq \frac{4(1-B)(1+\alpha)^{2}}{3(A-B)(1+2 \alpha)}\end{cases}
\end{aligned}
$$

where

$$
\gamma_{3}=\frac{4(1+\alpha)^{2} B}{3(1+2 \alpha)(A-B)}
$$

All the bounds are sharp.

Proof. From (1.7), we have

$$
f^{\prime}(z)+\alpha z f^{\prime \prime}(z)=\frac{1+A w(z)}{1+B w(z)} .
$$

Equating the coefficients of $z$ and $z^{2}$,

$$
a_{2}=\frac{(A-B)}{2(1+\alpha)} c_{1}
$$

and

$$
a_{3}=\frac{(A-B)}{3(1+2 \alpha)}\left(c_{2}-B c_{1}^{2}\right)
$$

So that

$$
a_{3}-\mu a_{2}^{2}=\frac{(A-B)}{3(1+2 \alpha)} c_{2}-\frac{(A-B)^{2}}{4(1+\alpha)^{2}}\left[\mu+\frac{4 B(1+\alpha)^{2}}{3(1+2 \alpha)(A-B)}\right] c_{1}^{2} .
$$

Therefore

$$
\left|a_{3}-\mu a_{2}^{2} \cdot\right| \leq \frac{(A-B)}{3(1+2 \alpha)}\left|c_{2}\right|+\frac{(A-B)^{2}}{4(1+\alpha)^{2}}\left|\mu+\frac{4 B(1+\alpha)^{2}}{3(1+2 \alpha)(A-B)}\right|\left|c_{1}\right|^{2}
$$

which together with (2.12) gives

$$
\left|a_{3}-\mu a_{2}^{2}\right| \leq \frac{(A-B)}{3(1+2 \alpha)}+\frac{(A-B)^{2}}{4(1+\alpha)^{2}}\left[\left|\mu+\gamma_{3}\right|-\frac{4(1+\alpha)^{2}}{3(1+2 \alpha)(A-B)}\right]\left|c_{1}\right|^{2}
$$

If $\left|\mu+\gamma_{3}\right| \leq \frac{4(1+\alpha)^{2}}{3(1+2 \alpha)(A-B)},(2.27)$ at once follows. 
If $\left|\mu+\gamma_{3}\right| \geq \frac{4(1+\alpha)^{2}}{3(1+2 \alpha)(A-B)}$, we get (2.30).

Consider the case when $\mu$ is real.

Case I. $\mu+\gamma_{3} \geq 0$.

(2.33) reduces to

$$
\begin{aligned}
\left|a_{3}-\mu a_{2}^{2}\right| & \leq \frac{(A-B)}{3(1+2 \alpha)}+\frac{(A-B)^{2}}{4(1+\alpha)^{2}}\left[\mu+\gamma_{3}-\frac{4(1+\alpha)^{2}}{3(1+2 \alpha)(A-B)}\right]\left|c_{1}\right|^{2} \\
& =\frac{(A-B)}{3(1+2 \alpha)}+\frac{(A-B)^{2}}{4(1+\alpha)^{2}}\left[\mu-\frac{4(1-B)(1+\alpha)^{2}}{3(1+2 \alpha)(A-B)}\right]\left|c_{1}\right|^{2},\left|c_{1}\right| \leq 1 .
\end{aligned}
$$

If $\mu \geq \frac{4(1-B)(1+\alpha)^{2}}{3(1+2 \alpha)(A-B)},(2.31)$ follows.

If $\mu \leq \frac{4(1-B)(1+\alpha)^{2}}{3(1+2 \alpha)(A-B)}$, we have

$$
\left|a_{3}-\mu a_{2}^{2}\right| \leq \frac{(A-B)}{3(1+2 \alpha)}
$$

Case II. $\mu+\gamma_{3} \leq 0$.

(2.33) takes the form

$$
\left|a_{3}-\mu a_{2}^{2}\right| \leq \frac{(A-B)}{3(1+2 \alpha)}-\frac{(A-B)^{2}}{4(1+\alpha)^{2}}\left[\mu+\frac{4(1+\alpha)^{2}(1+B)}{3(1+2 \alpha)(A-B)}\right]\left|c_{1}\right|^{2},\left|c_{1}\right| \leq 1
$$

If $\mu \leq \frac{-4(1+\alpha)^{2}(1+B)}{3(1+2 \alpha)(A-B)}$, we get (2.29).

Again

$$
\left|a_{3}-\mu a_{2}^{2}\right| \leq \frac{(A-B)}{3(1+2 \alpha)} \quad \text { if } \quad \frac{-4(1+\alpha)^{2}(1+B)}{3(1+2 \alpha)(A-B)} \leq \mu .
$$

(2.34) and (2.36) together yield (2.30).

Extremal function for (2.27) and (2.30) is given by

$$
\begin{aligned}
f_{0(3)}(z) & =P_{0}(z) * k_{0}(z) \text { where } \\
P_{0}(z) & =\int_{0}^{z} \frac{1+A t^{2}}{1+B t^{2}} d t
\end{aligned}
$$

and

$$
\begin{aligned}
k_{0}(z) & =\frac{1}{\alpha} z^{\left(\frac{1}{\alpha}\right)-1} \int_{0}^{z} \frac{t^{\left(\frac{1}{\alpha}\right)-1}}{1-t} d t \\
& =z+\sum_{k=2}^{\infty} \frac{1}{1+(k-1) \alpha} z^{k}
\end{aligned}
$$


Extremal functions for the bounds (2.28), (2.29) and (2.31) are given by

$$
\begin{aligned}
f_{1(3)}(z) & =P_{1}(z) * k_{0}(z) \text { where } \\
P_{1}(z) & =\int_{0}^{z} \frac{1+A t}{1+B t} d t .
\end{aligned}
$$

* denotes the convolution or Hadamard product of two functions.

The following theorem is an easy consequence of Theorem 2.4.

Theorem 2.5. If $f \in G(\alpha ; A, B)$, then

(i) for any complex number $\mu$,

$$
\left|a_{3}-\mu a_{2}^{2}\right| \leq \begin{cases}\frac{(A-B)}{(1+2 \alpha)}, & \left|\mu+\gamma_{4}\right| \leq \frac{(1+\alpha)^{2}}{(A-B)(1+2 \alpha)} \\ \frac{(A-B)^{2}}{(1+\alpha)^{2}}\left|\mu+\gamma_{4}\right|, & \left|\mu+\gamma_{4}\right| \geq \frac{(1+\alpha)^{2}}{(A-B)(1+2 \alpha)}\end{cases}
$$

and

(ii) for any real number $\mu$,

$$
\leq \begin{cases}\frac{-(A-B)^{2}\left(\mu+\gamma_{4}\right)}{(1+\alpha)^{2}}, & \mu \leq \frac{-(1+B)(1+\alpha)^{2}}{(A-B)(1+2 \alpha)} \\ \frac{(A-B)}{(1+2 \alpha)}, & \frac{-(1+B)(1+\alpha)^{2}}{(1+2 \alpha)(A-B)} \leq \mu \leq \frac{(1-B)(1+\alpha)^{2}}{(1+2 \alpha)(A-B)} \\ \frac{(A-B)^{2}}{(1+\alpha)^{2}}\left(\mu+\gamma_{4}\right), & \mu \geq \frac{(1-B)(1+\alpha)^{2}}{(A-B)(1+2 \alpha)}\end{cases}
$$

where

$$
\gamma_{4}=\frac{B(1+\alpha)^{2}}{(A-B)(1+2 \alpha)} .
$$

Extremal function $f_{0(4)}(z)$ for the bounds (2.38) and (2.41) is defined by

$$
f_{0(4)}(z)=z\left(\frac{1+A z^{2}}{1+B z^{2}}\right) * k_{0}(z) \text {. }
$$

Extremal function $f_{1(4)}(z)$ for the bounds (2.39), (2.40) and (2.42) is defined by

$$
f_{1(4)}(z)=z\left(\frac{1+A z}{1+B z}\right) * k_{0}(z)
$$




\section{References}

[1] H.S. Al-Amiri and M.O. Reade, "On a linear combinations of some expressions in the Theory of the univalent Functions," Monatshafte fur Mathematik 80(1975), 257-264.

[2] I.E. Bazilevic, "On the case of integrability in quadratures of the Lowner-Kufarev equation," Mat. Sb. 37(1955), 471-476.

[3] P.N. Chichra, "New sub-classes of the class of close-to-convex functions," Proc. Amer. Math. Soc. 4.2(1977), 37-43.

[4] R.M. El-Ashwah and D.K. Thomas, "Growth results for a sub-class of Bazilevic functions," Inter, J. Moth. and Math. Sci. 8(1985), 785-793.

[5] — "Some coefficient, length area results for a sub-class of Bazilevic function" (to appear).

[6] M. Fekete and G. Szegö, "Eine Bemerkung uber ungerade schlichte Funktionen," J. London Math. Soc. 8(1933), 85-89.

[7] R.M. Goel and Beant Singh Mehrok, "On the coefficients of a sub-class of starlike functions," Ind. J. Pure Appl. Math. 12(1981), 634-647.

[8] - "Some invariance properties of a sub-class of close-to-convex functions," Ind. J. Pure appl. Math. 12(1981), 1240-1249.

[9] —-, "A sub-class of univalent functions," J. A ust. Math. Soc. (Series A) 35(1983), 1-17.

[10] J. Hummel, "The coefficient regions of starlike functions," Pacific J. Math. 7(1957), 1381-1389.

[11] -, "Extremal problems in the class of starlike functions," Proc. Amer. Math. Soc. 11(1960), 741-749

[12] W. Janowski, "Some extremal problems for certain families of analytic functions," Ann. Polon, Math. 28(1973), 297-326.

[13] E.R. Keogh and E.P. Merkes, "A coefficient inequality for certain classes of analytic functions," Proc. Amer. Math. Soc. 20(1969), 8-12.

[14] S.S. Miller, P.T. Mocanu and M.O. Reade, "All $\alpha$-convex, functions are univalent and starlike," Proc. Amer. Math. Soc. 37(1973), 553-554.

[15] P.T. Mocanu, "Une propriete'de convexite' généralisée dans la théorie de la représentation conforme," Mathematica (CLUJ) 11(34)(1969), 127-133.

[16] R. Singh, "On Bazilevic functions," Proc. Amer. Math. Soc. 38(1973), 261-273.

[17] J. Szynal, "Some Remarks on Coefficients Inequality for $\alpha$-convex functions," Bull. De L'A cad. Des Sci Ser des Sci Math. Astr et phys. Vol. XX, No. 11(1972), 917-919.

[18] D.K. Thomas, "On a sub-class of Bazilevic functions," Inter. J. Math. and Math. Sci. 8(1985), 779-783.

Department of Mathematics, Punjabi University, Patiala - 147002, Punjab (India).

Department of Mathematics, 17, Khalsa College, Amritsar - 143002, Punjab (India). 